\title{
Non Volatile Low Power Wake up Radio Transceiver for Wireless Sensor Network
}

\author{
Vikas Kumar, Jagjit Singh, Arvind Kumar
}

\begin{abstract}
Wireless sensor nodes consume lots of energy during communication but huge power consumption has been observed during active listening in idle mode as source nodes can start data transmission at any time. Power saving can be achieved by establishing synchronization among end nodes. Many rendezvous solutions are available and out of which wake up receiver found extremely adroit. A non volatile wake up transceiver has been proposed in the present paper that works on the basis of ID matching. State of art using $4 G B$ of memory to remember states of sensor nodes while proposed technique used only 60 bits of memory with very less false alarm probability. Power consumption for proposed model is only $59.47 \mathrm{nW}$. Hence this model is quite effective in terms of power consumption and memory usage as compared to trailing models.
\end{abstract}

Index Terms: ID matching, low power, wake-up receiver, wireless sensor network

\section{INTRODUCTION}

Wireless sensor networks (WSNs) provide new technological elevation to many crucial applications like security, sports, entertainment and military etc. (Said, 2015). Most of sensor networks are battery operated and replacement or charging the power bank in application like space is very arduous. Energy efficiencies remain vital parameter for wireless sensor networks (Pflaum, Weigel, and Koelpin, 2018; .Lee, 2013). Power consumption during active communication is very huge and similarly huge power usufruct by sensor nodes while active listening in idle state (Pughat and Sharma, 2015). Therefore, the technique for judicially utilizing the power source is required and among state of arts available techniques wake-up receiver is rendering optimal result (Popovici, Magno and Marinkovic, 2013; Hutu et al.,2014). Incessant reform in design of wakeup receiver can create new realm for researchers. Elegant architecture for controlling switching states of sensor nodes can cut down usage of battery. In the present paper wake up receiver architecture is designed using a novel ID matching technique.

The proposed design can locate and manage switching of sensor nodes into different states like idle, active or sleep mode. Hash function operation will be performed on address bits of sensor node for parameters extraction and further atone with stored values in the memory as shown in Figure 1.

Revised Manuscript Received on June 15, 2019.

Vikas Kumar, Research Scholar, Department of ECE, IKGPTU, Kapurthala, Jalandhar, INDIA

Jagjit Singh, Associate Professor, Department of ECE, DAVIET, IKGPTU, Kapurthala, Jalandhar, INDIA

Arvind Kumar, Assistant Professor, Department of ECE, National Institute of Technology, Kurukshetra, INDIA

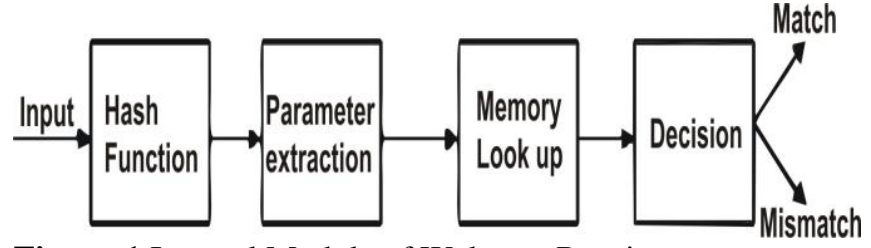

Figure-1 Internal Module of Wake-up Receiver

Wake up call has been initiated based upon atoning decision as shown in Figure 2. Present state of different nodes is stored in non volatile memory to avoid loss of states information in case of power failure.

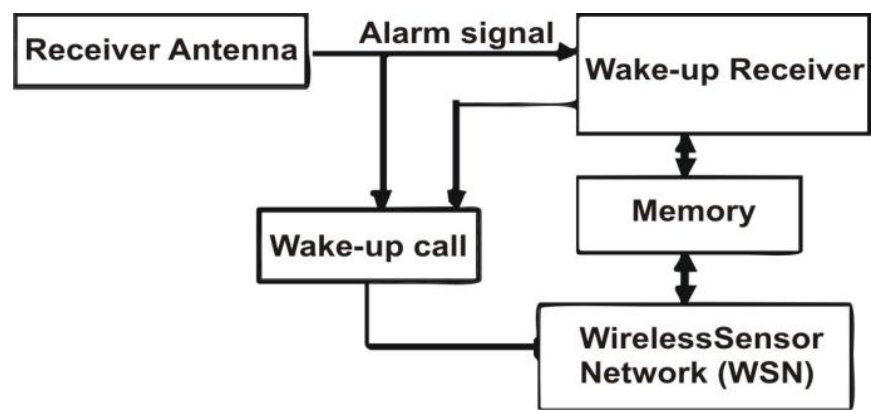

Figure-2 Architecture of ID matching Wake-up Receivers

In this way, proposed design can be utilized as general model in several WSN applications and further cost of receiver will be slashed by optimized use of non volatile memory.

\section{STATE OF ART}

This section presents the state of art about various techniques and aspects of low power design such as duty cycle control, improvement in battery, Network protocol and many aspects of Wake Up receiver (Berder and Sentieys, 2010).

\section{A. Battery Improvement}

With improvement in design and material used in battery may result in longer life. In this context, breakthrough has been achieved by using new discovered energy harvesting material which is composed of many substance i.e. NiMnCoIn (Wardlaw et al., 2013). It exhibits unique magnetic property that it can be used as transducer which can convert mechanical energy into magnetic field and further, into an alternating current (ac) with the help of special pick-up coil. Thus, generated alternating current can be converted into direct current and used as supply for wireless sensor 
nodes. In this way a self powered WSNs can be designed.

Another approach towards goal was proposed as energy efficient smart power unit by optimized use of super capacitor or utilizing Li-Ion battery for its maximum efficiency (Dikovic et al., 2011; Magno et al., 2012). But, such sort of solution can extend battery life by a small margin. Hence, this method is not the best solution.

\section{B. Duty cycle}

If the transceiver remains, in active state continuously then it draw continuously power from batteries even when there is no communication whereas cyclic fashion operation of transceiver becomes another viable option to reduce power consumption. This whole process is known as duty cycle control (Seyed et al., 2014). Present paper is focused on the second case and significant reduction in power usage has been observed. In duty cycle technique, receiver turns on in periodic fashion for short span of time (Magno and Benini, 2014). But, this solution is not the optimized solution as further delay trade off will come into play. Delay can be further reduced by using advanced software scheme which is used to manage the switching of receiver in wireless communication application (Stecklina et al., 2014). Low duty cycle protocols (LDCPs) can be used for efficiently implementations. LDCPs work on one time password technology but this communication is unreliable although energy consumption is not increasing.

\section{Network protocol and topology}

Routing protocol, topology control and scheduling of task can optimize energy in the field of communication model. Optimized Routing and Topology Optimisation Approaches has given significant improvement based on calculation of route path (Vodel et al., 2013). These approaches have given optimal results in case of asynchronous communication.

New clustering routing method i.e. predictive energy consumption efficiency (PECE) has been proposed for WSNs (Zhang et al., 2015). This protocol works in two stages: cluster formation and stable data transfer. In first stage, cluster has been formed using a complex algorithm which calculates shortest distance between nodes and their corresponding residual stage. In the second stage i.e. data can be transferred using PECE and bee colony optimization. High degree of improvement can be observed in overall network performance which reduces the energy consumption of whole network ultimately resulting in prolonging the survival time of the network.

SHEAR routing protocol monitor energy level of sensor nodes and selects the cluster head based on residual energy calculation (Shah et al., 2015).

The benefits of these protocols can be observed in term of evenly distribution of residual energy among all the nodes in clusters.

\section{Wake up receiver}

Wake up receiver is found as most promising among all available schemes. The idea of this type of receiver based on free space optical communication (FSO) principle (Mathew et al., 2009; 2010). Author justifies the use of a secondary, ultra low power wake-up channel by implementation of FSO transmission. The FSO channel is being used for wake up of receiver and high power radio can be used to transfer main data packets at high data rate. A reliable communication can be achieved in the range of fifteen meters with power consumption of $16.5 \mathrm{~mW}$ (Deng et al., 2012). Author also describe similar techniques in which laser has been used to send ID to unknown nodes for localisation of nodes in WSNs (Unterassinger et al., 2011).

Sensor nodes of WSN are idle during most of time but these access point (APs) in its idle state still consume a large percentage of power from battery (Tang et al., 2012). Hence, Wake-Up receiver presents a promising approach for minimizing the energy consumption in wireless communication environments to a greater extent. Sensor node have been turned on from sleep mode only in time of active data communication. RF energy detection and implementation of Wake-Up receiver result to low cost and run with low power consumption. As per IEEE 802.15.4 standard, before transmission of data, sender activate the RFID tag and RFID reader card has been installed at other end, on the interrupt pin of the node. In this way, transceiver can be migrated into deep sleep mode, during no transmission data packet (Jurdak et al., 2008; Gamm et al., 2010).

A problem of false triggering has been encountered during operation of Wake-Up receiver. Wake-Up receiver can be implemented using field-programmable gate array (FPGA) (Rosello and Riesgo, 2011; Uraiby et al. 2012). Asynchronous mode of communication reduces many overheads and battery usage days are increased after using of Wake Up receiver. One problem faced by most of researcher during design of Wake Up receiver is its volatile nature. In 5 bit ID code for nodes, memory requirement is 4GB (Vodel et al., 2012). States of different nodes can be stored and hence recently found solution has estimated power consumption of wake up receiver as $28.2 \mu \mathrm{W}$ (Shuangming et al., 2016). Low cost RF energy harvesting WSNs and developed model achieve a range of $16 \mathrm{~m}$ with $+13 \mathrm{~dB}$ transmitting power at data rate of 33.33kbps (Kaushik et al., 2016).

Verilog coding has been used for designing of transmitter and receiver (Divyabharti et al., 2013). In the same way, by using Verilog coding advance Wake Up receiver can also be designed for low power consumption.

\section{NUMERICAL ANALYSIS OF PROPOSED DESIGN}

The proposed design consists of two phases. In the first phase, network has been set up and address has been assigned to all nodes. Nodes are grouped into clusters based on parameter extraction. In second phase, power curb mechanism will be discussed

\section{A. Emplacement of wireless sensor networks}

In proposed architecture, membership function value can be evaluated after performing hashing on nodes ID and further cluster number can be calculated.

These calculated values must be updated in memory as unique identification in respect to node ID as explained below

Sensor node ID is unique sequence of binary numbers and can be delineate as

$$
X=\left[X 1_{2}, X 2, X 3_{i} \ldots \mathrm{Xn}\right]
$$

Where $\quad X n \in[0,1]$ 
Member function can be calculated with multiple hashing of nodes ID. Hash function $\mathrm{H}$ [i] can be delineate as

\section{$H k[i] \in f(x[i]]$}

Where it $\in[0,1,2, \ldots m]_{e} I$

In proposed architecture $\mathrm{k}$ number of distinct hash function are used

$$
\begin{gathered}
\mathrm{f}(\mathrm{X}[\mathrm{i}] \neq \mathrm{f}(\mathrm{X}[\mathrm{j}]) \\
\text { Where } \quad \mathrm{i}_{i} j \in\left[0,1_{2} 2_{2} \ldots \mathrm{m}\right]_{a} I
\end{gathered}
$$

Hence obtained value act as membership values and used to calculate cluster number for same sensor node.

Cluster No $=\sum_{i=2}^{k} H k[i]$

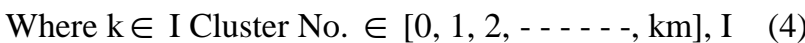
Total number of cluster are equal to k.m. Obtained values must also be updated in $\mathrm{m}$ bit memory. Present status of sensor nodes can be remembered with the aid of a non volatile memory in case of power failure or switch off mode.

\section{B. Power curb Mechanism}

Once the network has been established and all nodes have been added successfully in the respective clusters.

This phase describes how power can be saved with the help of implemented algorithm.

String of binary number has been transmitted. These transmitted bits acts as sensor node ID and hashed k- times similarly as done previously in emplacement phase.

\section{$H k[i] \in f(x[i])$}

Where $\quad \mathrm{i} \in[0,1,2 \mathrm{~m}]_{2} I$

Hence, in this way membership values have been calculated and reconciliation from non volatile memory will be carried out.

$$
\begin{aligned}
& f(x[i] \neq f(x[j]) \\
& \text { Where } I_{2} j \in\left[0,1_{2} 2_{2} \ldots \mathrm{m}\right]_{l} I
\end{aligned}
$$

If all values match, resulting to true alarm signal for transmitted sensor node ID which means request accepted and thus therein main communications can be started further. But if values mismatch, means access denied and sensor nodes will remain in deep sleep mode. In this way, false input can easily be detected.

Parallel processing has been used for further optimization, as $1^{\text {st }}$ mismatch of membership value detected, stop further calculation and false input has been reported for further action. Hence in this way, in both cases wake up receiver saves power and battery usage.

\section{DESIGNS AND SIMULATION RESULT}

Wake up receiver has been implemented and simulated using verilog. Figure 3 depicts the simulink model for wake-up receiver designed using Xilinx block set tool. Interfacing of Xilinx has been established with MATLAB with help of system generator.
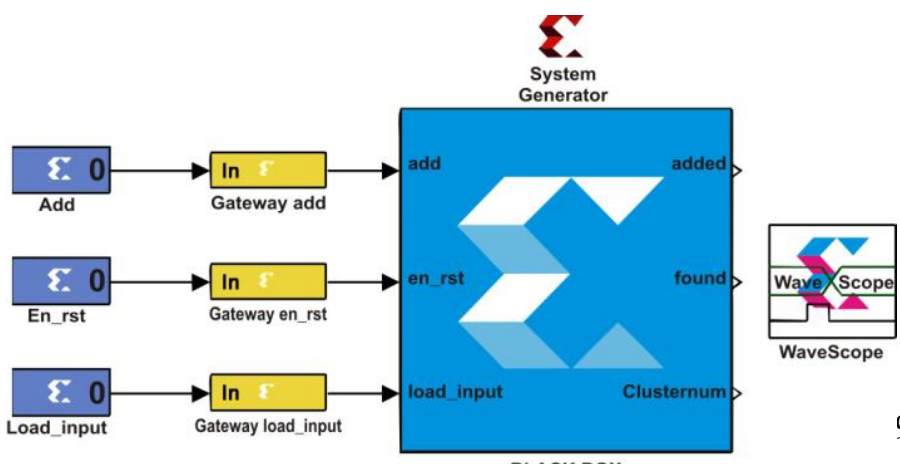

Figure-3 Model for Wake-up receiver

Designed model is simulated with below mentioned parameters

$\mathrm{n}$ ( Sensor node ID length $)=16$ bits

$\mathrm{k}$ ( Number of hash function) $=4$

$\mathrm{m}($ Memory bits used $)=60$ bits

Figure 4 depicts the transceiver model in simulink. It acts as 16 bits transmitter for designed system. These 16 bits of sensor node ID are transmitting through AWGN channel after modulation at transmitter end. At the receiver's end these codes are demodulated and decoded as shown in Figure 4. Received code acts as input for wake up receiver.

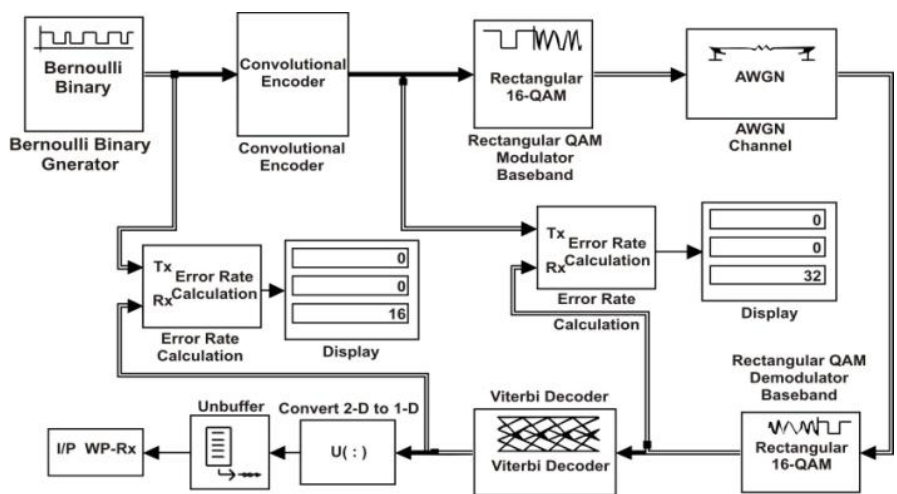

Figure-4 Transceiver simulink model

Output waveform as expected can be seen in figure 5 for three different casees.

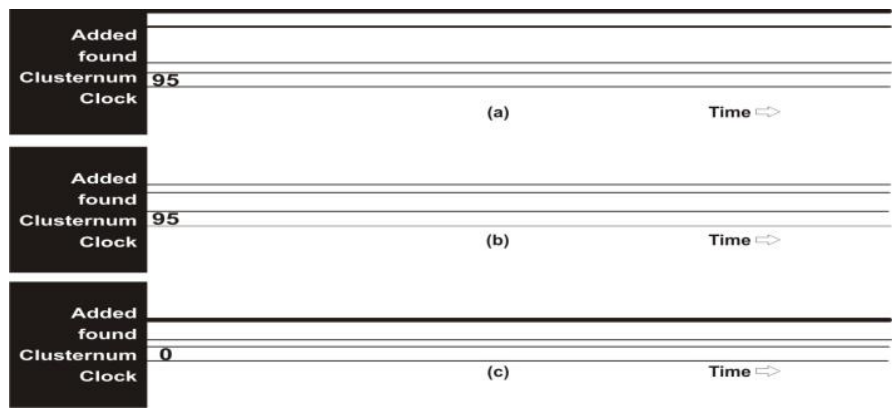

Figure 5 Output Waveform of Wake-up receiver at three different times

1. Waveform for new sensor node added into network Emplacement phase.

2. Sensor node accurately detected and instructed to start active communication with the help of main transceiver.

3. It shows waveform for power curb phase when called node does not exist in established network. All the output pins are at low voltage level. It shows the false input has been detected.

Hence in this way, lots of power can be saved and ultimately enhancement in battery life to a large extend.

There is trade off between BER and SNR. BER vs Eb/No have been plotted for proposed model and compared to theoretical graph for given dimensions in Figure 6. Proposed graph converge to zero early.

Published By BLACK BOX

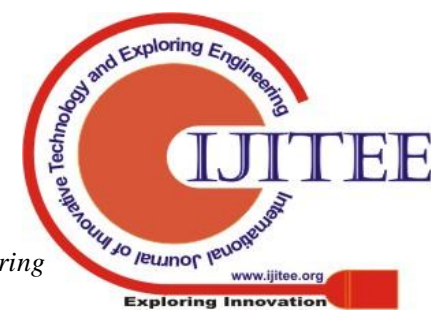




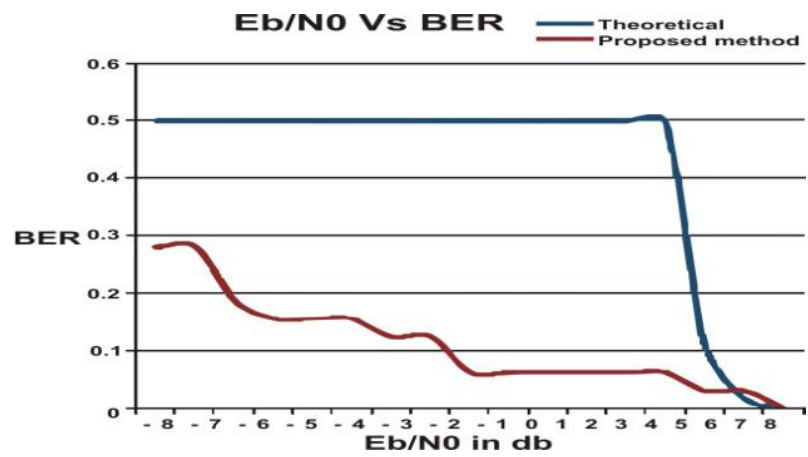

Figure $6 \mathrm{BER}$ vs $\mathrm{Eb} / \mathrm{No}$

Power consumption values have been analyzed using design compiler. Result has been found with technology link library "lsi_10k.db" and operating conditions is" WCCOM". Only 60 bits memory can serve the purpose with very less probability of fake alarm signal. The results so obtained have been showcased in the table 1 .

Table 1 Performance Summary and comparison table

\begin{tabular}{|l|l|l|l|}
\hline Parameter & $\begin{array}{l}\text { Proposed } \\
\text { work }\end{array}$ & $\begin{array}{l}\text { [Shuang } \\
\text { ming et } \\
\text { al.] }\end{array}$ & $\begin{array}{l}\text { [Kaushik } \\
\text { et al.] }\end{array}$ \\
\hline $\begin{array}{l}\text { Eb/No ( } \\
\text { Error free } \\
\text { transmission) }\end{array}$ & $+8 \mathrm{~dB}$ & NA & $+13 \mathrm{~dB}$ \\
\hline $\begin{array}{l}\text { Non volatile } \\
\text { Memory }\end{array}$ & 60 bits (yes) & $\begin{array}{l}4 \mathrm{~GB} \\
\text { (yes) }\end{array}$ & $(\mathrm{No})$ \\
\hline $\begin{array}{l}\text { Power } \\
\text { Consumption }\end{array}$ & $59.4658 \mathrm{nW}$ & $28.2 \mathrm{uW}$ & $\mathrm{NA}$ \\
\hline Data rate & $0.2 \mathrm{MHz}$ & $250 \mathrm{kbps}$ & $\mathrm{NA}$ \\
\hline
\end{tabular}

In Table 1, different performance parameters are compared with some latest papers and found that the proposed design provides the promosing results. Experiment demonstrates that by keeping transmitting power with an optimal range of $+8 \mathrm{~dB}$, an error free communication can be set up by using AWGN channel. However, the same results has been perceived by $+13 \mathrm{~dB}$ (Kaushik et al.2016). Moreover, 4 GB non volatile memory was used to store the sensor nodes states (Shuangming et al, 2016) however in present work, the same can be achieved with only 60 bits of memory. The proposed model is using only $59.46 \mathrm{nW}$ dynamic power, hence, Huge power saving is achieved.

\section{Conclusions}

Wake up receiver has been successfully implemented consuming very low power i.e. 59.46nW. The proposed receiver has been implemented with 16 bits of ID matching system which concludes that it can efficiently work on very large sensor network. Present model can be used in several applications of wireless sensor networks. Researcher will found its better use in various applications where frequent charging of battery is not possible. This model is more effective in term of power usage, bit error rate and security as compare to previous models. This model is also very beneficial in detecting false reception of node ID and much power will be saved in this process. It will also reduce false triggering of node with energy optimization.

\section{ACKNOWLEDGMENT}

The corresponding author would like to express gratitude to I.K. Gujral Punjab Technical University, Kapurthala, Jalandhar (Punjab) for providing infrastructural, computational and research facilities for pursuing research work.

\section{REFERENCES}

1. Said, O.," Performance evaluation of WSN management system for QOS gurantee," EURASIP Journal on Wireless Communications and Networking,2015, pp. 1-18

2. Pflaum,R., Weigel,R., and Koelpin, A., "Ultra-low-power sensor node with wake-up-functionality for smart-sensor-applications," 2018 IEEE Topical Conference on Wireless Sensors and Sensor Networks (WiSNet), Anaheim, CA, 2018, pp. 107-110.

3. Lee, D.," Fast notification architecture for wireless sensor network," International journal of electronics, Vol100, No.3, 2013, pp 371-383. http://dx.doi.org/10.1080/00207217.2012.713012

4. Pughat, A. and Sharma, V., "A review on stochastic approach for dynamic power management in wireless sensor network" Springer, Human-centric Computing and Information Sciences, 2015, pp $1-14$.

5. Popovici, E., Magno, M., and Marinkovic, S.," 'Power Management Techniques for Wireless Sensor Networks.” Advances in Sensors and Interfaces (IWASI), 2013 pp. 194-198

6. Hutu, F., Khoumeri, A., Villemaud, G., and Gorce , J.,”A new wake-up radio architecture for wireless sensor network" EURASIP Journal on Wireless Communications and Networking, 2013 pp.1-13

7. Berder, O., and Sentieys, O.,'Power optimized hardware/software framework for wireless motes," In Proc. of the Workshop on Ultra-Low Power Sensor Networks, Co-located with ARCS,2010, pp. 229-233

8. Wardlaw, J., Karaman, I., and Karsilayan, A.," Low-Power Circuits and Energy Harvesting for Structural Health Monitoring of Bridges," IEEE Sensors Journal, vol. 13, No. 2, 2013, pp.709-722

9. Dikovic, A., Sisul, G., and Modlic, B.," A Low Cost Platform For Sensor Network Applications and educational purposes," Radio engineering, Vol. 20, No. 4, 2011, pp. 758-765

10. Magno, M., Marinkovic, S., Brunelli, D., and Popovici, E.,’Smart Power Unit with Ultra Low Power Radio Trigger Capabilities for Wireless Sensor Networks," DATE '12 Proceedings of the Conference on Design, Automation and Test in Europe, 2012, pp. 75-80

11. Seyed, N., Mazloum, and Edfors, O., "Performance Analysis and Energy Optimization of Wake-Up Receiver Schemes for Wireless Low-Power Applications," IEEE Transaction on wireless communication, 2014, pp. 7050-7061.

12. Magno, M., and Benini, L.,"An Ultra Low Power High Sensitivity Wake-Up," IEEE Transactions on Industrial Informatics, 2014, pp. 92-99.

13. Stecklina, O., Kornemann, S., and Methfessel, M.,"A secure wake-up scheme for low power wireless sensor nodes," Collaboration Technologies and Systems (CTS) IEEE, 2014, pp.279-286, ISBN 978-1-4799-5157-4

14. Vodel, M., Lippmann, M., and Hardt, W.," Energy-Efficient Communication with Wake-Up Receiver Technologies and an Optimised Protocol Stack," International Conference on Advances in ICT for Emerging Regions, 2013, pp.177-184.

15. Zhang, D., Wang, X., Song, X., Zhang, T., and Zhu Y. ,'A new clustering routing method based on PECE for WSN," EURASIP Journal on Wireless Communications and Networking, 2015, pp 1-13, DOI: 10.1186/s13638-015-0399-x.

16. Ali Shah, M., Abbas, G., Basit Dogar, A. and Halim, Z. ," Scaling hierarchical clustering," Springer Complex Adapt System Model, 2015, pp. 1-23, DOI: 10.1186/s40294-015-0011-6.

17. Mathews, J., Barnes, M. and Arvind, D.,'Low Power Free Space," Optical Communication in Wireless Sensor Networks, Euro micro Conference on Digital System Design, 2009, pp. 849-856, DOI:10.1109/DSD.2009.234

18. Mathews, J., Barnes, M., Young, A., and Arvind, D.," Low Power Wake-Up in Wireless Sensor Networks using Free Space Optical Communications," International Conference 
on Sensor Technologies and Applications, 2010 pp. 257-261.

19. Deng, B., Li, W., Huang, G., Liu, S., and Zhang, Q.," High-accuracy and low-cost localisation scheme for wireless sensor networks," International Journal of Electronics, Vol. 99, No. 4, April 2012, pp 455-476, DOI: 10.1080/00207217.2011.609969

20. Unterassinger, H., Dielachery, M., Flatschery, M., Grubery, S., and Kowalczyky, G.,"A Power Management Unit for Ultra-Low Power Wireless Sensor Networks," IEEE Africon, 2011 pp. 1-6.

21. Tang S., Yomo H., Kondo Y., and Obana S. (2012), “ Wake-up receiver for radio-on-demand wireless LANs," EURASIP Journal on Wireless Communications and Networking, 2011, pp.1-13, DOI: 10.1186/1687-1499-2012-42.

22. Jurdak, R., Ruzzelli, A., and Hare, G., "Multi-hop RFID Wake-up Radio: Design Evaluation and Energy Tradeoffs," IEEE conference on Computer Communications and Networks, 2011, pp.1-8, DOI: 10.1109/ICCCN.2008.ECP.124.

23. Gamm, G., Sippel, M., Kostic M., and Reind, L.,"Low Power Wake-up Receiver for Wireless Sensor Nodes," IEEE International Conference on Intelligent Sensors, Sensor Networks and Information Processing, 2010, pp.121-126

24. Rosello, V., and Riesgo, T. ,'Ultra Low Power FPGA-Based Architecture for Wake-up Radio in Wireless Sensor Networks," Annual Conference on IEEE Industrial Electronics Society, 2011, pp. 3826-3831.

25. Uraiby, A., Yoshigoe, K., Seker, R., and Babiceanu, R. ,"FPGA Implementation of Low-profile Wake-up Radio Receiver for Wireless Sensor Networks," IEEE conference on consumer electronic, 2012, pp. 20-24

26. Vodel, M., Caspar, M., and Hardt, W. ,Wake-Up-Receiver Concepts -Capabilities and Limitations," Journal of networks, vol. 7, no. 1, 2012, pp 126-134.

27. Shuangming Y., Peng F., and Nanjian W. ," A low power non Volatile LR- WPAN baseband processor with wake up identification receiver " Communication system design, China Communications, 2016, pp 33-46.

28. Kaushik, K., Mishra, D., Swades De, Chowdhury, K., and Heinzelman, W.," Low cost Wake-up Receiver for RF Energy harvesting Wireless Sensor Networks," IEEE sensor, 2016 pp. 1-9, 2DOI: 10.1109/JSEN.2016.2574798

29. Divyabharathi, R., Scholar, P., Hakeem, C., and Mian, A.," Design and simulation of zigbee transmitter using verilog," International Conference on Information Communication and Embedded Systems, 2013, pp.882-888. international research publications to his credit and has authored 04 books..

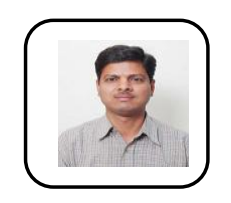

Dr. Arvind Kumar born in 1970. He has completed his B.Tech in 1996 from Allahabad University and M.Tech in 1999 from NIT Allahabad. He did his Ph.D. from NIT Kurukshetra, ECE department and working as Assistant Professor in the same department. His research interests include Wireless Communication, signal processing and communication system.

\section{AUTHORS PROFILE}

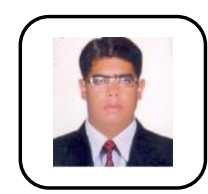

Mr. Vikas Kumar born in 1985. He did his B.Tech (ECE) from Kurukshetra university in 2006 and completed his M.Tech (VLSI design, ECE department) with distinguishing from NIT Kurukshetra in 2010. He is pursuing Ph.D. (Electronic Engineering department) from IKPTU Jalandhar, Punjab. His research interests include Low power design and Wireless sensor network.

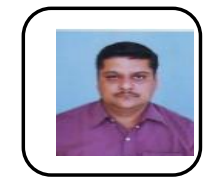

Dr. Jagjit Singh born in 1971 . He did his Ph.D. from PTU Jalandhar. He is a passionate researcher in the field of optical and wireless communication. He started his career as Lecturer in 1994 and is now working as Associate Professor in the department of electronics and communication engineering at DAV Institute of Engineering \& Technology, Jalandhar. His current research area is multichannel high capacity optical links and wireless networks. He has more than 35 number of 